\title{
An investigation of amino acid and acylcarnitine levels in neonates from the Tibet Autonomous
}

\section{Chunyan Zhang}

Chinese PLA General Hospital https://orcid.org/0000-0002-7582-2207

\section{Drun Dha}

The second people's hospital of Tibet Autonomous Region

\section{Yuxuan Cheng}

Chinese PLA General Hospital

\section{Ya Ma}

The second people's hospital of Tibet Autonomous Region

\section{Yan Meng}

Chinese PLA General Hospital

\section{Drun Tse}

The second people's hospital of Tibet Autonomous Region

\section{Dolma Ngawang}

Health commission of Tibet Autonomous Region

\section{Pedrun Dekyi}

Health commission of Tibet Autonomous Region

\section{Tao Jiang}

Chinese PLA General Hospital

\section{Yang Shu}

Chinese PLA General Hospital

\section{Jiayi Cui}

Chinese PLA General Hospital

\section{Jing Li}

Beijing Hospital

Yaping Tian ( $\sim$ tianyp@301hospital.com.cn )

https://orcid.org/0000-0002-8245-3745

\section{Research article}

Keywords: tandem mass spectrometry, Acyl carnitine, Reference interval, newborn screening

Posted Date: December 11th, 2019

DOI: https://doi.org/10.21203/rs.2.18538/v1 
License: (a) (i) This work is licensed under a Creative Commons Attribution 4.0 International License. Read Full License 


\section{Abstract}

Background: The purpose of the study was to establish reference values of amino acids and acylcarnitines in newborns of the Tibet Autonomous Region for the first time and to provide an experimental basis for the diagnosis of genetic metabolic diseases.

Methods: We detected concentrations of 43 kinds of amino acids, acylcarnitines and succinylacetone in the dried blood spots of 15029 newborns using liquid chromatography tandem mass spectrometry. We compared the indexes between Tibet and our lab, where most data come from an inland area and Han Chinese people. Then we compared amino acid and acylcarnitine levels of seven regions in Tibet and explored their impact factors. The distribution of amino acid and acylcarnitines were different in Tibet.

Results: Reference intervals of amino acids and acyl carnitines in neonates from the Tibet Autonomous Region were defined according to the (P 0.5\% P 99.5\%) of the values. Given the third reference range, the recall rate of statistical screening was significantly reduced to $2.16 \%$.

Conclusions: This study has contributed to the field by determining the actual values of amino acids and acylcarnitines in newborns from the Tibet Autonomous Region, which could be used as reference for a newborn metabolic screening project in this area.

\section{Introduction}

Inherited metabolic diseases (IMD) are caused by genetic mutations that interfere with typical metabolism. This genetic mutation tends to result in a deficiency of an enzyme defect, leading to a lack of the enzyme's products as well as an accumulation of the enzyme's substrates, which then causes a series of clinical symptoms [1]. At present, more than 1000 types of IMD have been diagnosed[2]. While individual metabolic disorders are rare, collectively, their incidence is approximately 1 in 1000 [3].

Owing to the high morbidity, mortality and strong risk of recurrence in affected families, neonatal IMD screening is an important element of modern preventive medicine as it allows the identification of both potential and asymptomatic infants as early as possible. Meanwhile, neonatal screening programs are an effective measure to reduce birth defects and improve the health of a population.

In the early 19th century, Millington [4] used liquid chromatography-tandem mass chromatography (LC$\mathrm{MS} / \mathrm{MS}$ ) in neonatal screening for the first time. Due to its high sensitivity, signal-to-noise ratio, high specificity and high selectivity, LC-MS/MS has become the ideal analysis technique for IMD screening [5] [6].In China, Shanghai and Zhejiang were the first to apply LC-MS/MS technology to newborn screening, and it is now being gradually introduced into the public health care network system in China [7] [8].

However, there is little data on neonatal IMD screening in the Tibet Autonomous Region. This region is located in the southwest of the Qinghai-Tibet Plateau. With a mean elevation of more than 4,000 meters, it is known as the 'roof of the world'. The region covers an area of 120.223 million square kilometers, which accounts for about one eighth of the total area of China. The Tibet Autonomous Region has 74 prefecture- 
level cities and had a population of approximately $300.220,000$, a birth rate of $15.39 \%$ and around 46,200 newborns in 2017. Statistics from the population and family planning commission of the region in 2013 showed that the incidence of birth defects in Tibet was significantly higher than the national average [9]. The 'birth defect intervention project' was started in this region in November 2015, meaning that neonatal IMD screening was carried out there for the first time.

We performed an investigation of amino acid and acylcarnitine levels in neonates from the Tibet Autonomous, with the aim of providing a preliminary reference range of amino acids and acylcarnitines using tandem mass spectrometry for newborns screening.

\section{Patients And Methods}

\section{Patients and Samples collection}

Samples were taken from 15029 live born infants from seven provinces of Tibet Autonomous Region between October 2015 and February 2019. Heel blood was collected after 72 hours after the infants were born and fully breastfed, and then dropped onto filter paper (Whatman 903). Blood spot samples were placed in the shade to dry and were sealed and stored at $-4^{\circ} \mathrm{C}$ prior to analysis.

\section{LC-MS/MS detection}

Small molecule metabolites of dry blood spots were extracted using underivatized amino acids, carnitine and succinylacetone assay kits (PerkinElmer, Finland) according to the manual. Concentrations of 43 types of amino acid, carnitine and succinylacetone were detected by liquid chromatography and tandem mass spectrometry (Xevo TQ Detector, Waters, Milford, USA) and then analyzed using the software Masslynx (Waters Corporation, Milford, USA) and Neolynx.(Waters Corporation, Milford, USA).

\section{Judgment criteria and intervene}

We recalled suspicious positive cases if the index was 1.5 times higher than the original blood specimen of the first screening. If the result of another check was still higher, parents were asked to test the urine organic acid and the infant's gene for a definite diagnosis.

Intervention and therapeutic schedules for the diseases were based on the clinic's norms of clinical genetic metabolic diseases, edited by Gu Xuefan. Follow ups allowed us to measure the therapy effect at intervals.

\section{Statistical Analysis}

Between-group comparisons were performed by independent-samples t-tests and ANOVA with Bonferroni posthoc multiple comparisons test for normally distributed data or Mann-Whitney U test for non-parametric 
sample distribution. Results from normally distributed data are expressed as Mean \pm SD while results of abnormally distributed data are expressed as Median $\left[P_{50}\left(P_{25}-P_{75}\right)\right]$. All statistical analysis was performed using SPSS (version 21.0), and two-tail $p<0.05$ was considered statistically significant. Graphs were generated using GraphPad Prism software (version 6.0).

\section{Results}

\section{The distribution of neonates from the Tibet Autonomous Region}

A total of 15029 neonatal screenings were conducted between 2016 and 2019, including 8576 males and 6453 females. Some $99.67 \%$ of these babies (14980/15029) were Tibetan. Our cases were distributed in seven prefecture-level cities in the Tibet Autonomous Region, including 4407 in Lhasa, 2726 in Naqu, 1164 in Changdu, 1082 in Shannan, 4974 in Xigaze, 308 in Ali and 368 in Nyingch. The distribution of neonates from the Tibet Autonomous Region is depicted in Table 1.

\section{Comparison to the accumulated laboratory results}

Before this study, there was little data on neonatal screening from the Tibet region and Tibetan people. As shown in Figure 1, concentration of many indexes in the Tibet region were different from our lab, where most data come from inland areas and Han Chinese people. Amino acids, ketones indexes, Arg, Phe, Cit and SA were higher, while Orn, Pro, Tyr, Gly, Leu+lle+Pro-OH and Val were lower in Tibet than in inland areas. The concentration of medium and long chain acylcarnitine C6-C18 were higher than in inland areas. If we recall a case with abnormal results, the misjudgment rate may be much higher and time of diagnosis would be extended.

\section{Preliminary establishment of the reference intervals of amino acids and acylcarnitines in neonates from the Tibet Autonomous Region}

We initially used the reference ranges of the laboratory-which were established by 100,000 healthy neonates data from an inland area-to judge results from the Tibet region and recall cases for reexamination and diagnosis according to the CLSI guideline [10]. Results of reexamination and diagnosis showed that the false positive rate was high, approximately $6 \%$, which was higher than the recall rate of $2 \%$ in other regions. A high recall rate brings an increased cost for diagnosis and additional distress for parents.

We therefore set 5000 cases as a node to establish the reference ranges suitable for screening in Tibet three times. Factors before blood sample collection, such as maternal feeding, nutritional supplement and gestational age were also considered, and cases with abnormal results in the recall test or proven disorders 
were excluded. Cases used to establish cutoff values were excluded from re-screening positive. The first reference range was determined when screening quantity reached 5000 , which would be used for following screening. The second range was established when screening quantity reached 10000 , and the third range was established when screening quantity reached 15000 . The reference intervals of amino acids and acylcarnitines in neonates from the Tibet Autonomous Region were determined according to the (P0.5\% P99.5\%) of the values(Table 2 and Supplemental Table). Based on the third reference range, the recall rate of statistical screening was significantly reduced to $2.16 \%$. These results, which can be seen in Table 3, are approximate to the recall rates of other regions.

\section{The effects of diet and altitude on small molecule metabolites}

The screening area covers seven prefecture-level cities. A total of $99.67 \%$ of the babies (14980/15029) were Tibetan. The mean blood collection time was 30 days after birth. Most babies had been given supplemental food from the birth, according to the local custom, which caused differences of small metabolites molecules between this area and inland areas. For example, babies in Xigaze region were fed by breast milk and zanba (highland barley) and were also given a small amount of barley wine and buttered tea. Therefore, we initially explored the impact of the special dietary habits in different areas of Tibet on the small molecule metabolites indicators.

As shown in Figure 2, many amino acids were significantly higher in Changdu and Naqu, where babies were fed with breast milk, cow (yak) milk and yoghurt, than in Lhasa, Xigaze and Shanan, where babies were fed with breast milk, zanba and yak butter tea. These results were replicated for lipometabolism levels. Although there was no significant difference in free carnitine $\mathrm{C} 0$ in the different regions, levels of shortchain acylcarnitine C2-C5 were higher in Changdu and Naqu and were lower in Ali, Xigaze and Shannan. Levels of medium and long chain acylcarnitine C6-C18 were significantly higher in Lhasa, Changdu, Nyingchi and Naqu than in Ali, Xigaze and Shannan.

In order to explore effects of climate and altitude on tandem results, we compared the results of Nyingchi and Ali, which have similar diets. Ali is located in the southwest border of China and has a mean altitude of more than $5000 \mathrm{~m}$. Due to its high altitude, the climate is cold and dry, annual rainfall is relatively low, the difference between day and night temperature is large, and winter is long and cold. In contrast, Nyingchi, which is located in the southeast of Tibet, has a mean altitude of $3100 \mathrm{~m}$, while its lowest point is only 900 $\mathrm{m}$, lower than other areas of Tibet. Due to the Indian ocean current, the climate is warm and comfortable. Annual rainfall is approximately $650 \mathrm{~mm}$, the average annual temperature is $8.7^{\circ} \mathrm{C}$, average annual sunshine is 2022.2 hours, and the frost-free period is 180 days. Therefore, while the two regions have similar population composition and eating habits, their altitudes, climates and other factors are different.

As shown in Figure 3, amino acids that easily degraded were lower in Nyingchi and higher in Ali region, such as Arg and Met. These results were consistent with previous reports that Arg and Met would fluctuate 
regularly, with low concentration in summer and high concentration in winter. Most acylcarnitine levels in the Ali region were lower in Ali, which may be related to the low basal metabolic rate in Ali region.

\section{Discussion}

In the past ten years, MS/MS was widely used for neonatal hereditary metabolic disease screening in China, improving the ability and efficiency of newborn screening [11]. However, until now, there was little data relating to the screening, diagnosis and monitoring of neonatal diseases in the Tibet Autonomous Region. This was for many reasons, such as nomadic and technological factors. Statistics from the population and family planning commission of the region in 2013 showed that incidences of birth defects in Tibet were significantly higher than the national mean. Therefore, early screening as well as timely and effective treatment are crucial for protecting the healthy growth of newborns from this area.

Prior to setting up the local reference interval, many laboratories around the country referred to the reference range of the new screening laboratory at Shanghai Xinhua hospital, where hundreds of thousands of newborns have been screened and thousands of suspected positive cases have been definite diagnosed. Small molecular metabolites reflect the conditions of newborns and mothers, while regional differences in dietary habits, ethnic groups, climate and incidence rates lead to regional differences in indicators. Therefore, it is necessary to establish the reference range of the local population, as this can reduce the false positive rate, reduce the recall rate and improve accuracy.

We aimed to investigate amino acid and acylcarnitine levels in neonates from the Tibet Autonomous Region. We analyzed samples from 15029 newborns in the Tibet region and established preliminary reference intervals for NBS screening in Tibet. Based on the current sequencing technology, we diagnosed eight cases with inherited metabolic diseases, four babies with phenylketonuria, one case of primary carnitine deficiency disorder, one case of tyrosinaemia type II, one case of short-chain acyl CoA dehydrogenase deficiency and one case medium-chain acyl CoA dehydrogenase deficiency.

Our data were mostly obtained from domestic provinces, including Beijing, Guizhou, Sichuan, Hubei and so on. Compared with inland newborn, the levels of Arg, Cit were higher and the level of Orn was lower in Tibetan newborn. These three aminos all participate in the urea cycle, which help cleanse amino acids metabolism toxins from your body. Under the action of enzyme, blood ammonia translates into carbamyl phosphate, combines with Orn and produce Cit, then generates Arg, and finally produce urea and Orn. Urea is eliminated from the body through the urine, and Orn re-enters the mitochondria, perpetuating the cycle [12] [13]. Therefore, these changes may be related to the dietary habits in Tibetan newborns, such as highprotein food, like yak milk. Interestingly, in the metabolism pathway of Phe and Tyr, Tibetan healthy newborn showed a higher level of Phe, and lower level of Tyr. Phe, one of special indexes for diagnosing hyperphenylalaninemia, had a maximum value $(95 \% \mathrm{Cl})$ of $130 \mu \mathrm{mol} / \mathrm{L}$ in Tibet, which is higher than our regular cutofff $120 \mu \mathrm{mol} / \mathrm{L}$ [14] [15]. The phenomenon maybe related to folate metabolism. It has been reported that a folate-increasing allele of the SNP rs1801133 at the MTHFR locus has an increased frequency in the Tibetan population, more than in Han population, which is possibly a consequence of adaptation to high UV radiation [16]. To illuminate the phenomenon, further research needed to be done. In 
terms of lipid metabolism, we observed that most acylcarnitine levels, especially medium and long chain acylcarnitine C6-C18, were higher than in inland area. C0 levels in babies from Tibet babies were lower than others. Newborns from the inland area may have a single diet source (milk or milk powder), but newborns in Tibet, due to local customs, have a varied diet. The distribution of amino acid and acylcarnitine was different in Tibet and reflect the difference of human metabolism activity in different regions.

We detected accuracy and inaccuracy before we performed the study to ensure that results were reliable. Our laboratory reported results of the neonatal genetic metabolic disease screening (tandem mass spectrometry technology) to the National Center for Examination and took part in interventricular quality assessment twice every year, which ensures that results are valid.

\section{Conclusion}

By examining data from more than 15,000 newborns, this study established a screening system for neonatal genetic metabolic diseases using tandem mass spectrometry in the Tibet Autonomous Region for the first time. Data reflect the metabolic level of newborns in Tibet and fill in gaps in the region as well as improving diagnostic efficiency for neonatal screening.

\section{Declarations}

\section{Ethics approval and consent to participate}

This study was approved by the Ethics Committee of the Chinese PLA General Hospital (No.S2018-025-01) and this trial has been verified by Chinese Clinical Trial Registry (No. ChiCTR1800016903). We obtained the informed written consent from all participants' parent or guardian for sample collection, as well as permission for the samples' use in research.

\section{Consent for publication}

The corresponding author and all of the authors have read and approved the final submitted manuscript.

\section{Availability of data and materials}

The datasets used and analysed during the current study are available from the corresponding author on reasonable request.

\section{Competing interests}

None of the authors had a personal or financial conflict of interest.

\section{Funding}

This research was supported by the National Key Research and Development Plan (2017YFC1001700), National Natural Science Foundation of China $(81741094,81501821)$ and Beijing Nova program (Z181100006218038). The funding 2017YFC1001700 and Z181100006218038 contributed to study design 
and sample collection, and the funding 81741094 and 81501821 contributed to data analysis and interpretation.

\section{Authors' contributions}

ZCY, DD and TYP designed the study; DD, MYa, TD, ND, DP and LJ took charge of organizing nurse to recruit the patients and collect the samples. CYX, JT and SY preformed the clinical test; MYan and CJY were responsible for clinical recall and diagnosis; ZCY and TYP analyzed the data and wrote the manuscript.

\section{Acknowledgments}

Authors thank the support and salvage from the March of Dimes Birth Defects Foundation of China. The authors are also very grateful to the health committee of Nagqu district, Xigaze district, Shannan district, Qamdo district, Ali district, Nyingchi district for their energetic support.

\section{Abbreviations}

IMD, Inherited metabolic diseases; Ala, Alanine; Arg, Arginine; Cit, Citrulline; Gly, glycine; Leu, Leucine; Ile, isoleucine; Pro-OH, hydroxyprolin; Met, methionine; Orn, ornithine; Phe, phenylalanine; Pro, proline; $\mathrm{SA}_{2} ; \mathrm{CO}$, free carnitine; C2, Acetyl carnitine; C3, Propionyl carnitine; C3-DC, Malonyl carnitine; C4, Butyryl/isobutyryl carnitine; C4-DC, Methylmalonyl/succinyl carnitine; C5:1, Tiglyl carnitine; C5, Isovaleryl carnitine; C5-DC, Glutaryl carnitine; $\mathrm{C5}-\mathrm{OH}, 3-H y d r o x y$-isovaleryl carnitine; C6, Hexanoyl carnitine; C6-DC, Adipoyl carnitine; C8:1, Octenoyl carnitine; C8, Octanoyl carnitine; C10:1, Decenoyl carnitine; C10, Decanoyl carnitine; C12:1, Dodecenoyl carnitine; C12, Dodecanoyl carnitine; $\mathrm{C12}-\mathrm{OH}$, 3-Hydroxydodecanoyl carnitine; C14:2, Tetradecadienoyl carnitine; C14:1, Tetradecenoyl carnitine; C14, Tetradecanoyl carnitine; C14:1-OH, 3Hydroxy-tetradecenoyl carnitine; $\mathrm{C} 14-\mathrm{OH}$, 3-Hydroxy-tetradecanoyl carnitine; $\mathrm{C} 16: 1$, Palmitoleoyl carnitine;

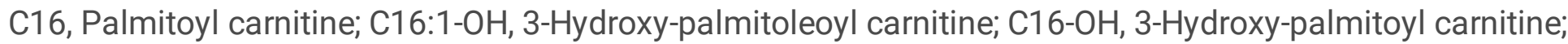
C18:2, Linoleyl carnitine; C18:1, Oleoyl carnitine; C18, Stearoyl carnitine; C18:2-OH, 3- Hydroxy-linoleyl carnitine; $\mathrm{C} 18: 1-\mathrm{OH}, 3-\mathrm{Hydroxy}$-oleoyl carnitine.

\section{References}

1.Sahai I, Marsden D: Newborn screening. Crit Rev Clin Lab Sci 2009, 46(2):55-82.

2.Ferreira CR, van Karnebeek CDM: Inborn errors of metabolism. Handb Clin Neurol 2019, 162:449-481.

3.Hendriksz CJ: Inborn errors of metabolism for the diagnostic radiologist. Pediatr Radiol 2009, 39(3):211220.

4.Flygare S, Wikstrom P, Johansson G, Larsson PO: Magnetic aqueous two-phase separation in preparative applications. Enzyme Microb Technol 1990, 12(2):95-103.

5.Rashed MS, Bucknall MP, Little D, Awad A, Jacob M, Alamoudi M, Alwattar M, Ozand PT: Screening blood spots for inborn errors of metabolism by electrospray tandem mass spectrometry with a microplate batch 
process and a computer algorithm for automated flagging of abnormal profiles. Clin Chem 1997, 43(7):1129-1141.

6.Tu W, Song X, Dai F, Ho JJ: Application of liquid chromatography-tandem mass spectrometry (LC-MS/MS) in screening of high risk children with inherited metabolic diseases in northern China. J Pediatr Endocrinol Metab 2010, 23(12):1245-1252.

7.Gu X, Zhou J, Ye J: [Neonatal screening for congenital adrenal hyperplasia in Shanghai areas]. Zhonghua Yu Fang Yi Xue Za Zhi 2002, 36(1):16-18.

8.Maitusong R, Japaer R, Zhao ZY, Yang RL, Huang XL, Mao HQ: Newborn screening in Zhejiang, China. Chin Med J (Engl) 2012, 125(4):702-704.

9.Cui Z, Wu F, Li Y, Xiao D, Chen R: [Survey on birth defects related knowledge among women of childbearing age in Tibetan autonomous region]. Zhonghua Yu Fang Yi Xue Za Zhi 2015, 49(6):576-578.

10.Morris Mh, gary; Cerda, Blas; Chace, Donald;Copeland, Sara; Morrissey, Mark; Stanley, Eleanor; Barshop, Bruce; George, M; Halim, Abdel-Basel; Hannon, William; Hooper, Pleasant; Litsheim, Thomas; Pasquali, Marzia; Poston, Philip; Whitley, Ronald: CLSI. Newborn Screening by Tandem Mass Spectrometry. Clinical and Laboratory Standards Institute 2010.

11.Huang X, Zhang Y, Hong F, Zheng J, Yang J, Tong F, Mao H, Huang X, Zhou X, Yang R et al: [Screening for amino acid metabolic disorders of newborns in Zhejiang province:prevalence, outcome and follow-up]. Zhejiang Da Xue Xue Bao Yi Xue Ban 2017, 46(3):233-239.

12.Ali EZ, Zakaria Y, Mohd Radzi MA, Ngu LH, Jusoh SA: Mutation Study of Malaysian Patients with Ornithine Transcarbamylase Deficiency: Clinical, Molecular, and Bioinformatics Analyses of Two Novel Missense Mutations of the OTC Gene. Biomed Res Int 2018, 2018:4320831.

13.Kiykim E, Zubarioglu T, Cansever MS, Celkan T, Haberle J, Aktuglu Zeybek AC: Coagulation Disturbances in Patients with Argininemia. Acta Haematol 2018, 140(4):221-225.

14.Li N, Jia H, Liu Z, Tao J, Chen S, Li X, Deng Y, Jin X, Song J, Zhang L et al: Molecular characterisation of phenylketonuria in a Chinese mainland population using next-generation sequencing. Sci Rep 2015, 5:15769.

15.Liu N, Huang Q, Li Q, Zhao D, Li X, Cui L, Bai Y, Feng Y, Kong X: Spectrum of PAH gene variants among a population of Han Chinese patients with phenylketonuria from northern China. BMC Med Genet 2017, 18(1):108.

16.Yang J, Jin ZB, Chen J, Huang XF, Li XM, Liang YB, Mao JY, Chen X, Zheng Z, Bakshi A et al: Genetic signatures of high-altitude adaptation in Tibetans. Proc Natl Acad Sci U S A 2017, 114(16):4189-4194.

\section{Tables}


Table 1. Distribution of neonates from the Tibet Autonomous Region

\begin{tabular}{|c|c|c|c|c|c|c|c|}
\hline \multirow[t]{2}{*}{ District } & \multicolumn{3}{|c|}{ Gender } & \multicolumn{2}{|c|}{ Gestational age } & \multicolumn{2}{|c|}{ Birth weight } \\
\hline & Male,n/\% & $\begin{array}{c}\text { Female } \\
, \mathrm{n} / \%\end{array}$ & total & Average & $95 \% \mathrm{CI}$ & Average & $95 \% \mathrm{CI}$ \\
\hline Lhasa & $2557 / 58$ & $1850 / 42$ & 2557 & 39.51 & $\begin{array}{c}39.45- \\
39.57\end{array}$ & 3202.32 & $\begin{array}{l}3174.96- \\
3229.67\end{array}$ \\
\hline Ali & $164 / 53$ & $144 / 47$ & 164 & 39.75 & $\begin{array}{c}39.64- \\
39.86\end{array}$ & 4172.18 & $\begin{array}{c}3987.09 \\
4357.28\end{array}$ \\
\hline Xigaze & $2911 / 59$ & $2063 / 41$ & 2911 & 39.25 & $\begin{array}{c}39.21- \\
39.29\end{array}$ & 3291.78 & $\begin{array}{l}3257.51- \\
3326.05\end{array}$ \\
\hline Linzhi & $214 / 58$ & $154 / 42$ & 214 & 38.97 & $\begin{array}{c}38.66- \\
39.29\end{array}$ & 3224.50 & $\begin{array}{c}3127.11- \\
3321.90\end{array}$ \\
\hline Changdu & $651 / 56$ & $513 / 44$ & 651 & 39.44 & $\begin{array}{l}39.37- \\
39.51\end{array}$ & 3408.72 & $\begin{array}{c}3345.79- \\
3471.66\end{array}$ \\
\hline Shannan & $589 / 54$ & $493 / 46$ & 589 & 39.82 & $\begin{array}{c}39.76- \\
39.88\end{array}$ & 3178.86 & $\begin{array}{l}3100.46- \\
3257.26\end{array}$ \\
\hline Naqu & $1490 / 55$ & $1236 / 45$ & 1490 & 39.27 & $\begin{array}{c}39.19- \\
39.35\end{array}$ & 3468.35 & $\begin{array}{c}3406.55- \\
3530.14\end{array}$ \\
\hline Total & $8576 / 57$ & $6453 / 44$ & 15029 & 39.37 & $\begin{array}{c}39.34- \\
39.40\end{array}$ & 3322.69 & $\begin{array}{c}3301.75- \\
3343.63\end{array}$ \\
\hline
\end{tabular}

Table 2. Statistical results of amino acids and acylcarnitines of neonates from the Tibet Autonomous Region 
$\begin{array}{llllllll}\text { (umol/L) } & \mathrm{P}, 0.001 & \mathrm{P}, 0.005 & \mathrm{P}, 0.01 & \mathrm{P}, 0.5 & \mathrm{P}, 0.99 & \mathrm{P}, 0.995 & \mathrm{P}, 0.999\end{array}$ $\begin{array}{lllllllll}\text { ALA } & 303.84 & 84.98 & 118.80 & 132.25 & 285.17 & 658.42 & 723.03 & 905.09\end{array}$

\begin{tabular}{lllllllll} 
ARG & 19.78 & 1.26 & 1.50 & 1.71 & 17.37 & 61.38 & 68.49 & 84.47 \\
CIT & 18.22 & 3.87 & 5.19 & 5.86 & 14.89 & 66.25 & 75.76 & 97.65 \\
\hline
\end{tabular}

GLY

$\begin{array}{llllllll}366.31 & 83.62 & 114.73 & 127.81 & 328.27 & 999.44 & 1093.36 & 1507.33\end{array}$

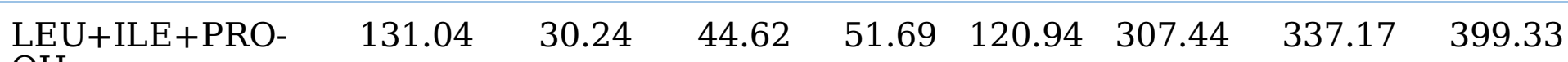
$\mathrm{OH}$

\begin{tabular}{lrrrrrrrr}
\hline MET & 12.96 & 2.14 & 2.55 & 2.96 & 11.94 & 32.50 & 35.85 & 45.43 \\
\hline ORN & 96.60 & 10.57 & 17.12 & 20.37 & 79.04 & 352.47 & 411.44 & 540.01 \\
\hline PHE & 47.98 & 11.39 & 18.77 & 21.23 & 44.95 & 103.41 & 113.05 & 134.65 \\
\hline PRO & 166.43 & 36.65 & 61.43 & 70.63 & 157.55 & 347.15 & 378.98 & 468.79 \\
\hline SA & 0.68 & 0.28 & 0.34 & 0.38 & 0.66 & 1.08 & 1.14 & 1.27 \\
\hline TYR & 80.72 & 17.78 & 28.29 & 31.78 & 74.05 & 190.46 & 207.98 & 268.92 \\
\hline VAL & 135.51 & 32.96 & 49.11 & 55.19 & 124.34 & 326.05 & 366.33 & 430.67 \\
\hline C0 & 29.97 & 6.92 & 8.44 & 9.56 & 28.24 & 66.22 & 72.29 & 84.06 \\
\hline C2 & 12.31 & 0.45 & 0.84 & 1.12 & 10.93 & 36.63 & 40.72 & 49.74 \\
\hline C3 & 1.48 & 0.14 & 0.20 & 0.26 & 1.30 & 4.28 & 4.86 & 6.07 \\
\hline C3DC+C4OH & 0.08 & 0.01 & 0.01 & 0.01 & 0.06 & 0.27 & 0.30 & 0.39 \\
\hline C4 & 0.21 & 0.07 & 0.08 & 0.08 & 0.19 & 0.47 & 0.53 & 0.66 \\
\hline C4DC+C5OH & 0.21 & 0.06 & 0.07 & 0.08 & 0.20 & 0.48 & 0.52 & 0.63 \\
\hline C5 & 0.14 & 0.02 & 0.03 & 0.04 & 0.12 & 0.39 & 0.44 & 0.58 \\
\hline C5:1 & 0.01 & 0.00 & 0.00 & 0.00 & 0.01 & 0.02 & 0.02 & 0.03 \\
\hline C5DC+C6OH & 0.12 & 0.02 & 0.03 & 0.04 & 0.11 & 0.28 & 0.32 & 0.42 \\
\hline C6 & 0.04 & 0.01 & 0.01 & 0.01 & 0.04 & 0.09 & 0.10 & 0.13 \\
\hline C6DC & 0.07 & 0.02 & 0.02 & 0.02 & 0.06 & 0.19 & 0.22 & 0.46 \\
\hline C8 & 0.05 & 0.01 & 0.01 & 0.01 & 0.05 & 0.13 & 0.14 & 0.19 \\
\hline C8:1 & 0.13 & 0.01 & 0.02 & 0.02 & 0.11 & 0.40 & 0.44 & 0.54 \\
\hline C10 & 0.01 & 0.01 & 0.01 & 0.06 & 0.20 & 0.23 & 0.31 \\
\hline C10:1 & 0.01 & 0.01 & 0.01 & 0.04 & 0.13 & 0.15 & 0.18 \\
\hline & & & & & & & & 0.03 \\
\hline
\end{tabular}




\begin{tabular}{lllllllll}
$\mathrm{C} 10: 2$ & 0.01 & 0.00 & 0.00 & 0.00 & 0.01 & 0.04 & 0.05 & 0.09 \\
\hline $\mathrm{C} 12$ & 0.06 & 0.01 & 0.01 & 0.01 & 0.05 & 0.20 & 0.23 & 0.31 \\
\hline $\mathrm{C} 12: 1$ & 0.04 & 0.00 & 0.01 & 0.01 & 0.03 & 0.15 & 0.18 & 0.25 \\
\hline $\mathrm{C} 14$ & 0.16 & 0.02 & 0.03 & 0.04 & 0.15 & 0.38 & 0.42 & 0.50 \\
\hline $\mathrm{C} 14: 1$ & 0.05 & 0.01 & 0.01 & 0.01 & 0.04 & 0.17 & 0.20 & 0.29 \\
\hline $\mathrm{C} 14: 2$ & 0.01 & 0.00 & 0.00 & 0.00 & 0.01 & 0.03 & 0.04 & 0.05 \\
\hline $\mathrm{C} 14 \mathrm{OH}$ & 0.01 & 0.00 & 0.00 & 0.00 & 0.01 & 0.02 & 0.03 & 0.03 \\
\hline $\mathrm{C} 16$ & 1.75 & 0.23 & 0.32 & 0.38 & 1.39 & 5.24 & 5.67 & 6.80 \\
\hline $\mathrm{C} 16: 1$ & 0.10 & 0.01 & 0.02 & 0.02 & 0.07 & 0.36 & 0.39 & 0.48 \\
\hline $\mathrm{C} 16: 1 \mathrm{OH}$ & 0.10 & 0.01 & 0.02 & 0.02 & 0.08 & 0.30 & 0.34 & 0.45 \\
\hline $\mathrm{C} 16 \mathrm{OH}$ & 0.02 & 0.00 & 0.00 & 0.01 & 0.01 & 0.04 & 0.04 & 0.05 \\
\hline $\mathrm{C} 18$ & 0.72 & 0.12 & 0.16 & 0.19 & 0.67 & 1.70 & 1.84 & 2.15 \\
\hline $\mathrm{C} 18: 1$ & 1.13 & 0.20 & 0.27 & 0.32 & 1.08 & 2.39 & 2.63 & 3.12 \\
\hline $\mathrm{C} 18: 1 \mathrm{OH}$ & 0.02 & 0.00 & 0.01 & 0.01 & 0.02 & 0.05 & 0.06 & 0.07 \\
\hline $\mathrm{C} 18: 2$ & 0.21 & 0.03 & 0.04 & 0.05 & 0.19 & 0.56 & 0.61 & 0.80 \\
\hline $\mathrm{C} 18 \mathrm{OH}$ & 0.01 & 0.00 & 0.00 & 0.00 & 0.01 & 0.02 & 0.02 & 0.02 \\
\hline
\end{tabular}

Table 3. Statistics based on the third reference range of tandem screening in Tibet 


\begin{tabular}{|c|c|c|c|c|c|c|c|}
\hline \multirow[t]{2}{*}{ District } & \multicolumn{4}{|c|}{ MS/MS results ( $\mu \mathrm{mol} / \mathrm{L})$} & \multirow[t]{2}{*}{ Total } & \multicolumn{2}{|c|}{ Statistics } \\
\hline & Nornal & $\mathrm{n} / \%$ & Abnornal & $\mathrm{n} / \%$ & & $X^{2}$ & $P$ value \\
\hline Lhasa & 4369 & $99.14 \%$ & 38 & $0.86 \%$ & 4407 & 95.390 & 0.000 \\
\hline Naqu & 2631 & $96.52 \%$ & 95 & $3.48 \%$ & 2726 & & \\
\hline Changdu & 1110 & $95.36 \%$ & 54 & $4.64 \%$ & 1164 & & \\
\hline Shannan & 1067 & $98.61 \%$ & 15 & $1.39 \%$ & 1082 & & \\
\hline Xigaze & 4865 & $97.81 \%$ & 109 & $2.19 \%$ & 4974 & & \\
\hline Ali & 301 & $97.73 \%$ & 7 & $2.27 \%$ & 308 & & \\
\hline Linzhi & 362 & $98.37 \%$ & 6 & $1.63 \%$ & 368 & & \\
\hline Total & 14705 & $97.84 \%$ & 324 & $2.16 \%$ & 15029 & & \\
\hline
\end{tabular}

\section{Figures}
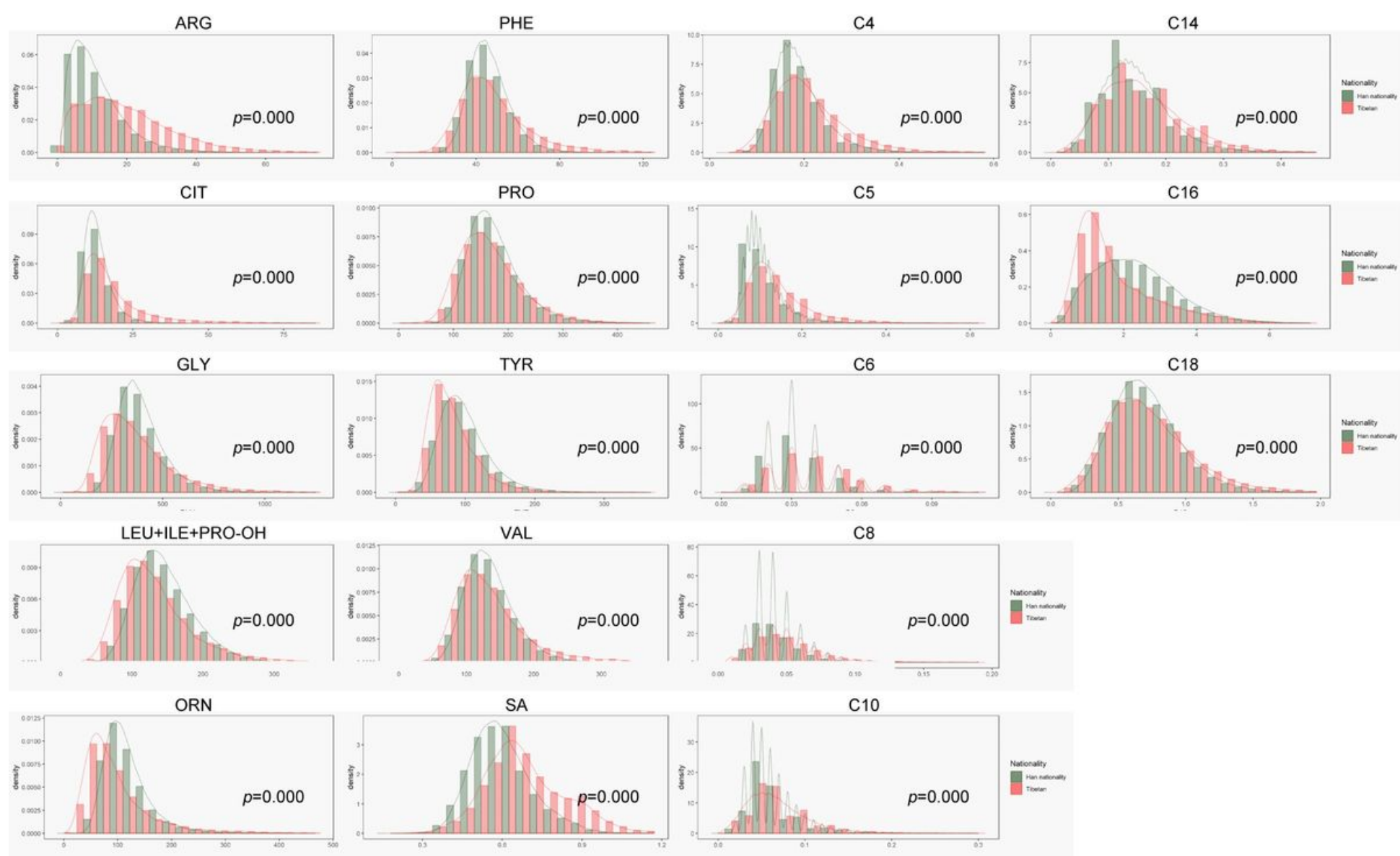

Figure 1 


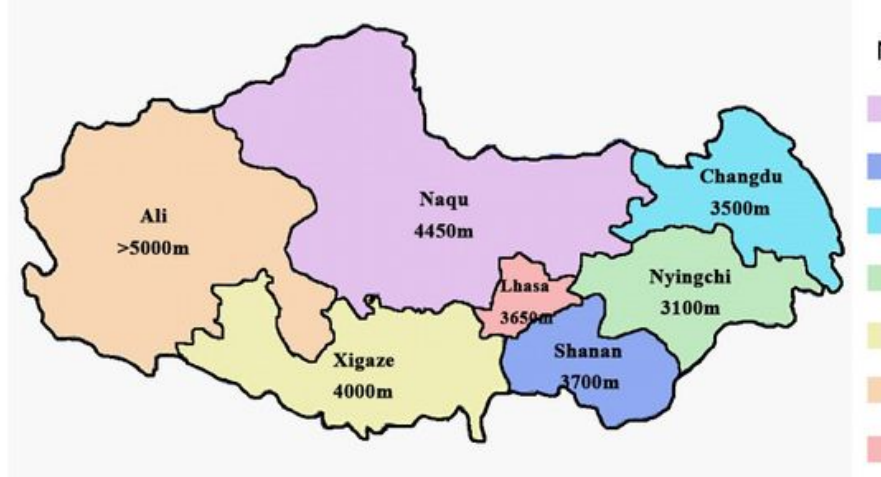

ARG

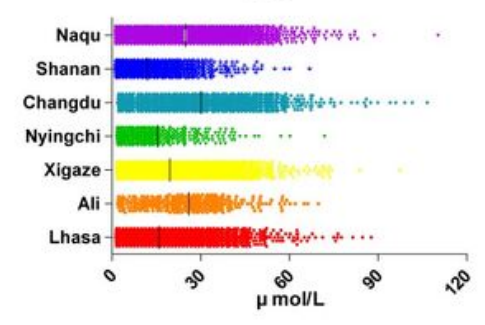

PHE

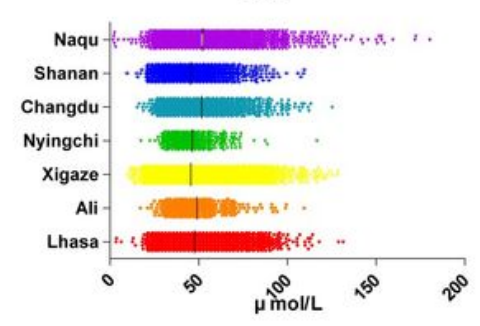

C3

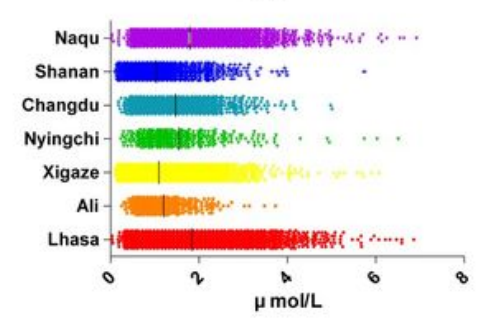

C10

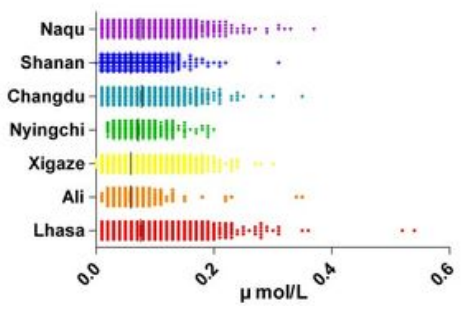

CIT

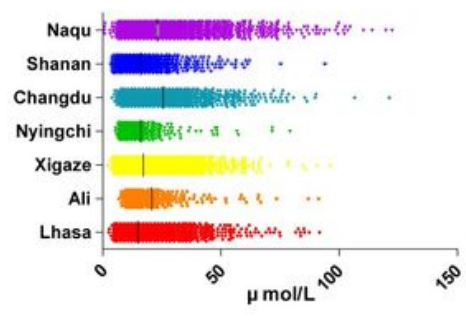

TYR

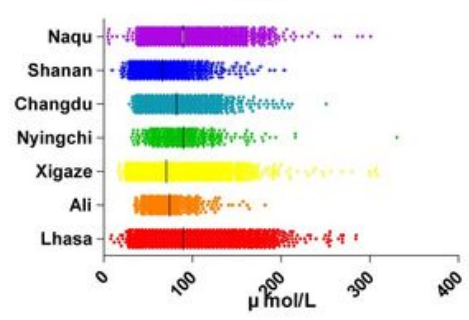

C4

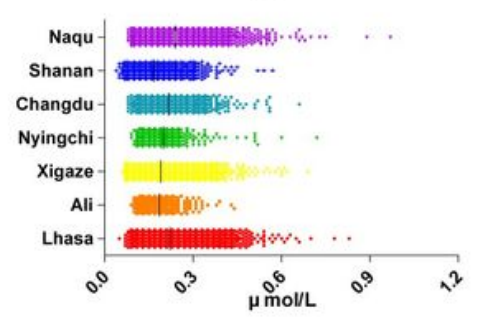

C14

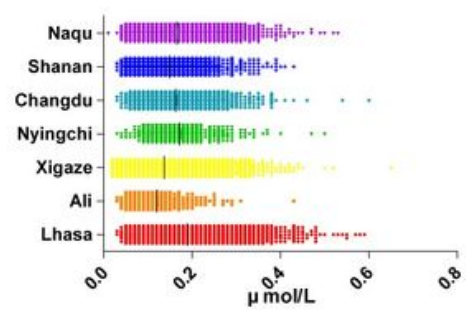

Neonatal Diet

breast milk, cow (yak) milk, yogurt

breast milk, zanba, butter tea

milk, cow (yak) milk, yogurt

breast milk, cow (yak) milk, zanba

breast milk, zanba, barley wine, buttered tea

breast milk, cow (yak) milk, ghee tea

breast milk, zanba (highland barley), yak butter tea

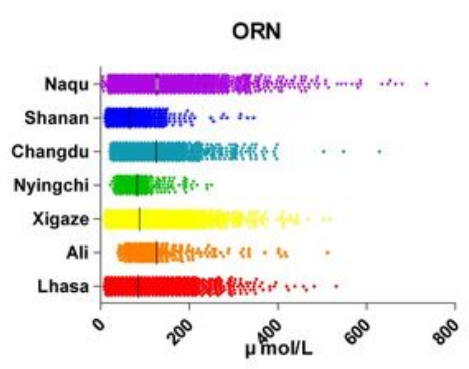

LEU+ILE+PRO-OH

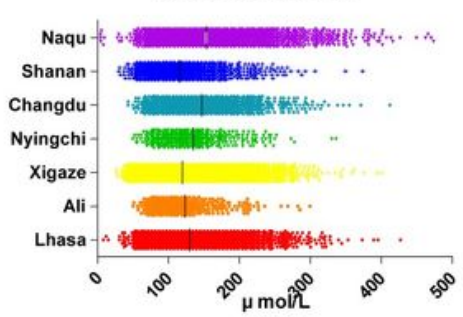

C5

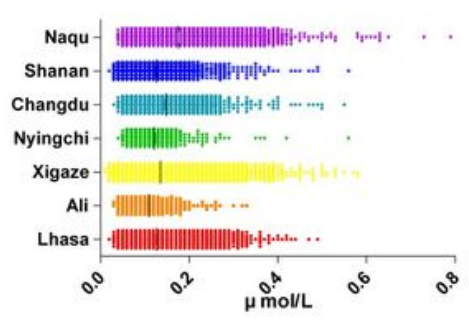

C18

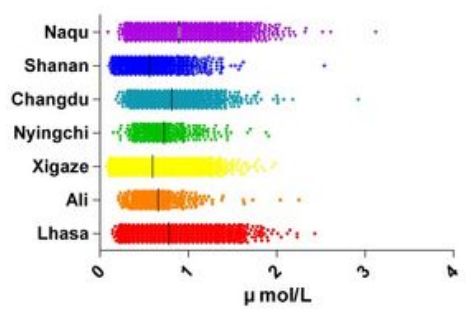

\section{Figure 2}

Neonatal diet in Tibet Autonomous Region as well as amino acids and acylcarnitine in different areas of Tibet 
ARG

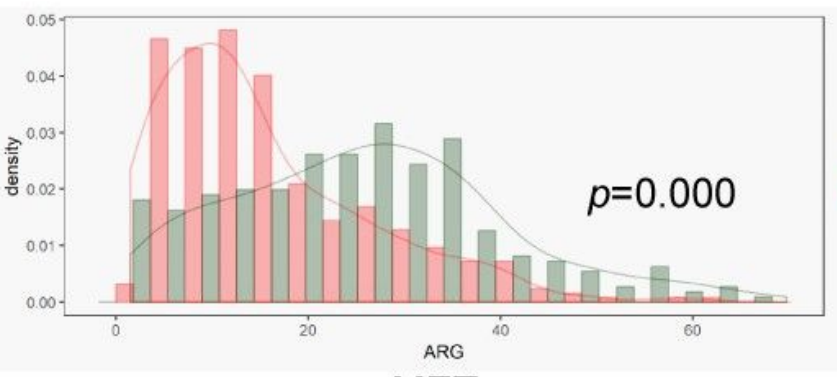

MET

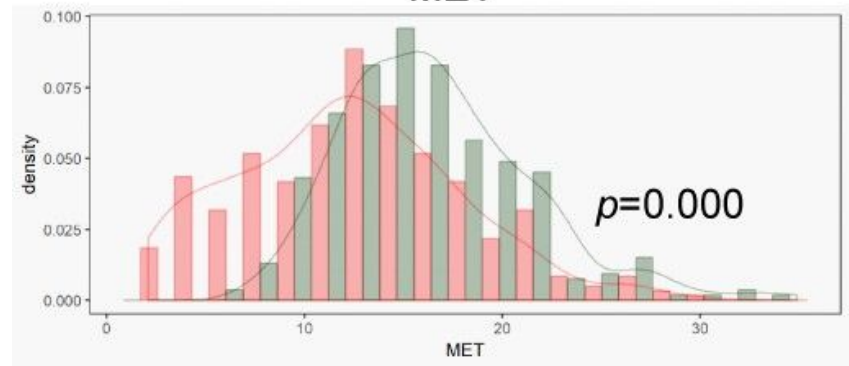

Co
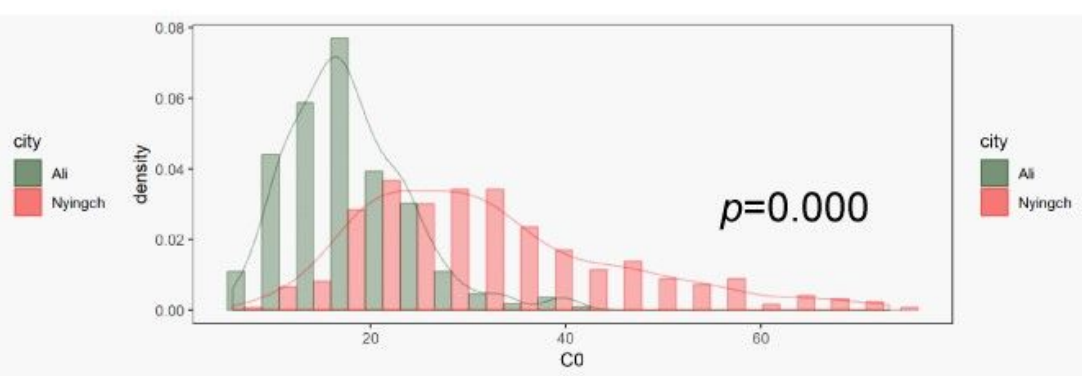

C16

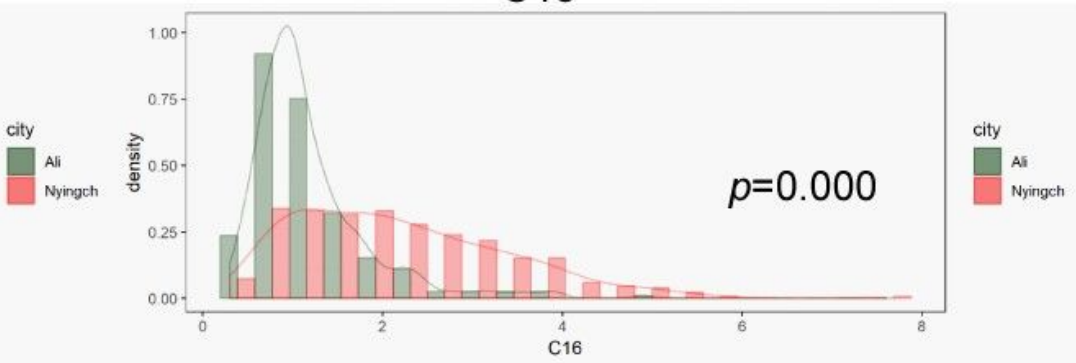

Figure 3

Comparison of metabolite index between Ali and Nyingchi

\section{Supplementary Files}

This is a list of supplementary files associated with this preprint. Click to download.

- SupplementalTable.docx 